\title{
Claudin gene expression profiles and clinical value in colorectal tumors classified according to their molecular subtype
}

This article was published in the following Dove Medical Press journal: Cancer Management and Research

\section{Sara Cherradi \\ Pierre Martineau \\ Céline Gongora \\ Maguy Del Rio}

Institut de Recherche en Cancérologie de Montpellier (IRCM), INSERM UI I 94, Université de Montpellier, Institut régional du Cancer de Montpellier, Montpellier F-34298, France
Correspondence: Maguy Del Rio Institut de Recherche en Cancérologie de Montpellier (IRCM), INSERM UI I 94 -ICM, 208 Avenue des Apothicaires, F-34298 Montpellier Cedex 5, France $\mathrm{Tel}+33467612416$

Email maguy.delrio@icm.unicancer.fr
Purpose: Colorectal cancer (CRC) is a heterogeneous disease that can be classified into distinct molecular subtypes. The aims of this study were 1) to compare claudin (CLDN) gene expression in CRC samples and normal colon mucosa, and then in the different CRC molecular subtypes, and 2) to assess their prognostic value.

Patients and methods: $C L D N$ expression in CRC samples was analyzed using gene expression data for a cohort of 143 primary CRC samples, and compared in the same CRC samples classified into different molecular subtypes (C1 to C6 according to the Marisa's classification, and CMS1 to CMS4 of the consensus classification). Comparison of CLDN expression in normal and tumor colon samples was also made on a smaller number of samples. Then, the relationship between $C L D N$ expression profiles and overall survival (OS) and progression-free survival was examined.

Results: Compared with normal mucosa, CLDN1 and CLDN2 were upregulated, whereas CLDN5, 7, 8, and 23 were downregulated in CRC samples. Variations in CLDN expression profiles were observed mainly in the $\mathrm{CMS} 2 / \mathrm{C} 1$ and $\mathrm{CMS} 4 / \mathrm{C} 4$ subtypes. Overall, expression of $C L D N 2$ or $C L D N 4$ alone had a strong prognostic value that increased when they were associated. In the CMS4/C4 subtypes, lower expressions of CLDN11, CLDN12, and CLDN23 were associated with longer OS. Conversely, in the CMS2 and C1 subtypes, low CLDN23 expression was associated with shorter OS and progression-free survival, suggesting a dual role for CLDN23 as a tumor suppressor/promoter in CRC. CLDN6 and CLDN11 had a prognostic value in the CMS2 and C4 subtypes, respectively.

Conclusion: This analysis of $C L D N$ gene expression profiles and prognostic value in CRC samples classified according to their molecular subtype shows that CRC heterogeneity must be taken into account when assessing $C L D N$ potential value as prognostic markers or therapeutic targets.

Keywords: colon, cancer, classification, prognosis, claudin, target, tight junction, heterogeneity

\section{Introduction}

Colorectal cancer (CRC) originates from the oncogenic transformation of the intestinal epithelium that physiologically acts as a functional barrier between the intestinal mucosa and the luminal environment. It is now becoming clear that epithelial cell polarity is a major gatekeeper against cancer initiation and metastasis formation. ${ }^{1}$ Epithelial cell polarity depends on the establishment of the apical junctional complex that includes tight junctions (TJs) and adherens junctions. ${ }^{2} \mathrm{TJ}$, the most apical of these intercellular junctions, play an essential role in maintaining cell polarity and in 
the regulation of paracellular permeability. ${ }^{3}$ Alterations in TJs by downregulation or upregulation of $\mathrm{TJ}$ proteins can trigger malignant transformation and influence cancer progression. ${ }^{4}$

Claudins (CLDNs) are TJ core components ${ }^{5}$ that are essential for TJ formation ${ }^{6}$ and contribute to their selectivity. ${ }^{7}$ In mammals, the CLDN family includes 27 members divided into two groups: classic and nonclassic CLDNs. ${ }^{8}$ Almost all CLDNs have a short intracellular N-terminal domain, four transmembrane domains, two extracellular loops, and an intracellular C-terminal domain that contains a PDZ-domainbinding motif for linking to TJ-associated proteins, such as MUPP1, PATJ, ZO-1, ZO-2 and ZO-3, MAGUKs, PAR3, PAR6, and PALS..$^{910}$ These proteins function as adaptors at the cytoplasmic surface of TJ strands and can directly or indirectly interact with cytosolic and nuclear proteins, for instance cytoskeletal molecules, regulatory proteins, tumor suppressors, and transcription factors. ${ }^{11}$ Finally, some CLDNs interact with cell adhesion proteins (eg, EPCAM) or receptors (eg, EPHA and EPHB). ${ }^{12}$ The cytoplasmic tail of most CLDNs contains a large number of predicted phosphorylation sites that could be involved in molecular interactions. ${ }^{13}$ Accumulated evidence indicates that CLDNs are associated with various pathways, including the WNT/ $\beta$-catenin, JAK-STAT3, and Notch signaling cascades. ${ }^{7,13,14}$

CLDNs are expressed in a cell- and tissue-specific manner. In the intestine, CLDNs display specific spatiotemporal expression profiles with variations along the crypt-lumen axis. ${ }^{15}$ Their expression can be regulated by various mechanisms at the transcriptional or posttranscriptional level, but also via mRNA stability modulation ${ }^{16}$ and through epigenetic mechanisms. ${ }^{16-19}$

CLDN expression is altered in several cancer types in a tumor-specific manner, and can vary according to the tumor stage. ${ }^{11}$ CLDN aberrant expression in tumors may have opposite functions (promotion of tumorigenesis and metastasis formation, or suppressive effects). ${ }^{13,20}$ For example, CLDN-1 is a cancer invasion/metastasis suppressor in lung adenocarcinoma, ${ }^{21}$ while in CRC, CLDN1 expression enhances the invasive ability and metastatic properties. ${ }^{22}$ Moreover, some CLDNs have an important regulatory role in the epithelial-mesenchymal transition (EMT) ${ }^{23-25}$ CLDNs can also serve as a hub for different signaling proteins, and therefore could have a critical role in the regulation of carcinogenesis or cancer progression. ${ }^{26}$ Finally, CLDN expression has been associated with patient survival, suggesting that they could be prognostic markers and/or therapeutic targets..$^{14,27,28}$

In CRC, research has focused mainly on CLDN1, 22,28,29 CLDN2, ${ }^{18,30,31}$ and $C L D N 7 .{ }^{24,32,33}$ Changes in the expression of TJ-related genes, including CLDNs, have been reported in CRC; ${ }^{34,35}$ however, these studies did not consider CRC heterogeneity, which can be described using molecular subtypes based on gene expression profiles. ${ }^{36-39}$

In this study, $C L D N$ gene expression profiles were investigated in a cohort of 143 primary CRC samples classified according to their molecular subtype and for which gene expression and clinical data were available. ${ }^{40-42}$ The expression of each $C L D N$ gene was first compared in normal and tumor colon samples, and then among the different CRC molecular subtypes. Finally, the prognostic value of the different expression profiles was evaluated.

\section{Patients and methods Gene expression analysis}

In this study, expression data for tumor samples from 143 patients coming from three cohorts (REG/P, ${ }^{40}$ COSIVAL, and BIOCOLON ${ }^{41,42}$ ) were used. These three studies were approved by the relevant ethics committees and all participants were informed about the study, and they signed a written informed consent before enrolment. All patients selected for this study had metastatic colorectal cancer (mCRC), and did not receive any chemotherapy treatment before primary tumor resection. Colon samples (normal colon, primary tumor, and hepatic metastasis samples from the REG/P cohort, and only primary tumor specimens from the COSIVAL and BIOCOLON cohorts) were collected at the time of surgery, following a standardized procedure to obtain high-quality RNA. ${ }^{43}$ Samples were then hybridized to human genome U133 Plus 2.0 arrays (Affymetrix Inc., Santa Clara, CA, USA). The gene expression data can be found online at the Gene Expression Omnibus under the accession numbers GSE62080 and GSE72970.

All 143 CRC samples were classified using the molecular classifications based on gene expression profiles that have been proposed by Marisa et $\mathrm{a}^{136}$ and Guinney et a ${ }^{39}$ (Table 1), as described in each reference publication. ${ }^{42}$ Briefly, Marisa et al described six molecular subtypes ( $\mathrm{C} 1$ to $\mathrm{C} 6$ ) with the following main features: $\mathrm{C} 1=\mathrm{CIN}$ and immune pathway downregulation; $\mathrm{C} 2=\mathrm{MSI} ; \mathrm{C} 3=$ mutated KRAS; $\mathrm{C} 4=$ stem cell phenotype-like; $\mathrm{C} 5=\mathrm{CIN}$ and upregulation of the WNT pathways; and $\mathrm{C} 6=\mathrm{CIN}$ and normal-like gene expression profile. ${ }^{36}$ The consensus classification includes four subtypes: CMS1 (microsatellite instability [MSI]-immune), CMS2 (epithelial and canonical), CMS3 (epithelial and metabolic), and CMS4 (mesenchymal). Most of the MSI-high tumors belong to the CMS1 subtype that has the best survival without recurrence. Chromosomal instability (CIN) tumors have a 
Table I Distribution of patients with $\mathrm{mCRC}$ according to the tumor molecular subtype

\begin{tabular}{|l|l|l|}
\hline Subtypes & $\mathbf{N}=\mathbf{I 4 3}$ & $\%$ \\
\hline Marisa $(\mathrm{n}=1 \mathbf{3 8})$ & & \\
\hline $\mathrm{Cl}$ & 27 & 19.6 \\
\hline $\mathrm{C} 2$ & 17 & 12.3 \\
\hline C3 & 19 & 13.8 \\
\hline C4 & 20 & 14.5 \\
\hline C5 & 36 & 26.1 \\
\hline C6 & 19 & 13.8 \\
\hline Missing & 5 & \\
\hline Consensus ( $\mathrm{n}=104)$ & & \\
\hline CMSI & 15 & 14.4 \\
\hline CMS2 & 29 & 27.9 \\
\hline CMS3 & 24 & 23.1 \\
\hline CMS4 & 36 & 34.6 \\
\hline Missing & 39 & \\
\hline
\end{tabular}

Abbreviation: $\mathrm{mCRC}$, metastatic colorectal cancer.

more heterogeneous gene expression pattern and, therefore, can be classified from CMS2 to CMS4. CMS4 tumors have a significantly higher risk of distant relapse. ${ }^{38,39}$

The Tsuji cohort (GSE28702) (4 $^{44}$ includes 83 stage IV CRC samples from patients treated with the FOLFOX regimen. Dr Shingo Tsuji kindly provided the overall survival (OS) data for this series.

\section{Statistical analyses}

For gene expression analyses, differences between groups were determined using the Kruskal-Wallis/Dunn's test.

Progression-free survival (PFS) was defined as the time from the beginning of first-line treatment for mCRC until recurrence or death. Alive patients without progression were censored at the date of last contact. OS was calculated from the beginning of first-line treatment until death. Correlations between $C L D N$ gene expression and PFS or OS were evaluated in the entire group ( $\mathrm{n}=143$ patients) and according to the tumor molecular subtype. In each subtype, CRC samples were divided into two groups (high/low expression) based on the median $C L D N$ gene expression. The Kaplan-Meier method was used to compare PFS and OS values, and the log-rank test was used to assess differences between survival distributions. For all experiments, differences were considered to be significant when $P<0.05$.

\section{Results}

\section{CLDN gene expression patterns in colon}

Analysis of the $C L D N$ gene expression levels from Affymetrix data for 17 normal colon mucosa, 20 primary CRC samples, and 19 hepatic metastases (REG/P cohort) ${ }^{40,41}$ showed that $8 C L D N$ genes (CLDN6, 9, 10, 11, 14, 16, 17, and 18 ) were weakly expressed in all samples. Among the other ten claudins, $C L D N 1, C L D N 3, C L D N 4$, and $C L D N 7$ were strongly expressed in primary CRC and metastatic samples, and $C L D N 3, C L D N 4, C L D N 7, C L D N 8$, and CLDN23 in normal mucosa (Figure 1A).

To determine whether $C L D N$ gene expression levels change during tumorigenesis, $C L D N$ expression profiles in normal mucosa, primary tumor, and hepatic metastases were compared. CLDN4, 6, 9, 10, 12, 14, 15, 16, 17, and 18 displayed similar expression levels in all tissue samples, whereas $C L D N 3$ and $C L D N 11$ showed a tendency to downregulation in primary tumor samples (Figure S1). On the other hand, $C L D N 1$ and $C L D N 2$ were significantly upregulated, and $C L D N 5,7,8$, and 23 downregulated in primary tumor and hepatic metastasis samples compared with normal mucosa (Figure 1B). These results were validated in 15 matched samples from the study cohort (normal mucosa and primary tumor from the same patient; Figure S2). The largest variation of expression between paired samples was observed for $C L D N 2$ and $C L D N 8$.

\section{Comparison of CLDN gene expression in the different CRC molecular subtypes}

$C L D N$ expression was then analyzed in all 143 primary CRC samples classified in molecular subtypes according to Marisa et al ( $\mathrm{C} 1$ to $\mathrm{C} 6)$ and the consensus classification (CMS1 to 4; see Table 1 and Figure 2 for the relationships between classifications). ${ }^{42}$

Among the $C L D N$ genes that were upregulated in tumors compared with normal mucosa, CLDN1 expression was significantly higher in the CMS2 subtype and in the $\mathrm{C} 1$ and C5 subtypes, while CLDN2 expression was similar in all subtypes (Figure 3A and Figure S3A). Among the CLDN genes downregulated in tumors, $C L D N 8$ expression reduction was less pronounced in the CMS3 and C6 subtypes, while $C L D N 7$ and $C L D N 23$ were more downregulated in the CMS4 and C4 subtypes (Figure 3B and Figure S3B). Among the $C L D N$ genes with comparable expression in normal and tumor tissues, $C L D N 3$ and $C L D N 4$ showed lower expressions in the CMS4 subtype (not significant for CLDN4) and in the $\mathrm{C} 4$ subtype (Figure $3 \mathrm{C}$ and Figure $\mathrm{S} 3 \mathrm{C}$ ). Among the $C L D N$ genes that were weakly expressed in primary tumors, $C L D N 6$ expression level was higher in the CMS2 and $\mathrm{C} 1$ subtypes, and CLDN11 expression was strongest in the CMS4 and $\mathrm{C} 4$ subtypes (Figure $3 \mathrm{C}$ and Figure $\mathrm{S} 3 \mathrm{C}$ ). The other $C L D N$ genes did not show any significant expression level difference among CRC subtypes. 
A

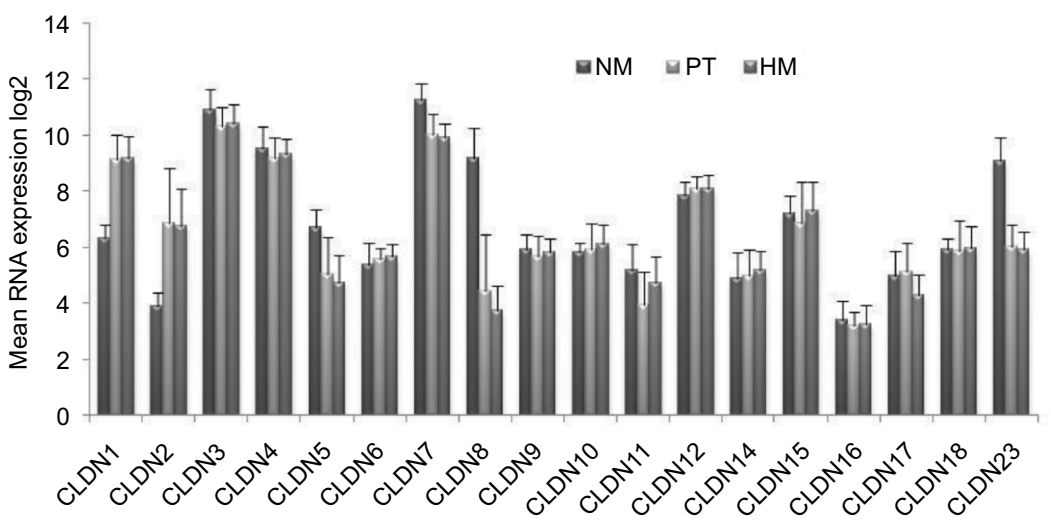

B
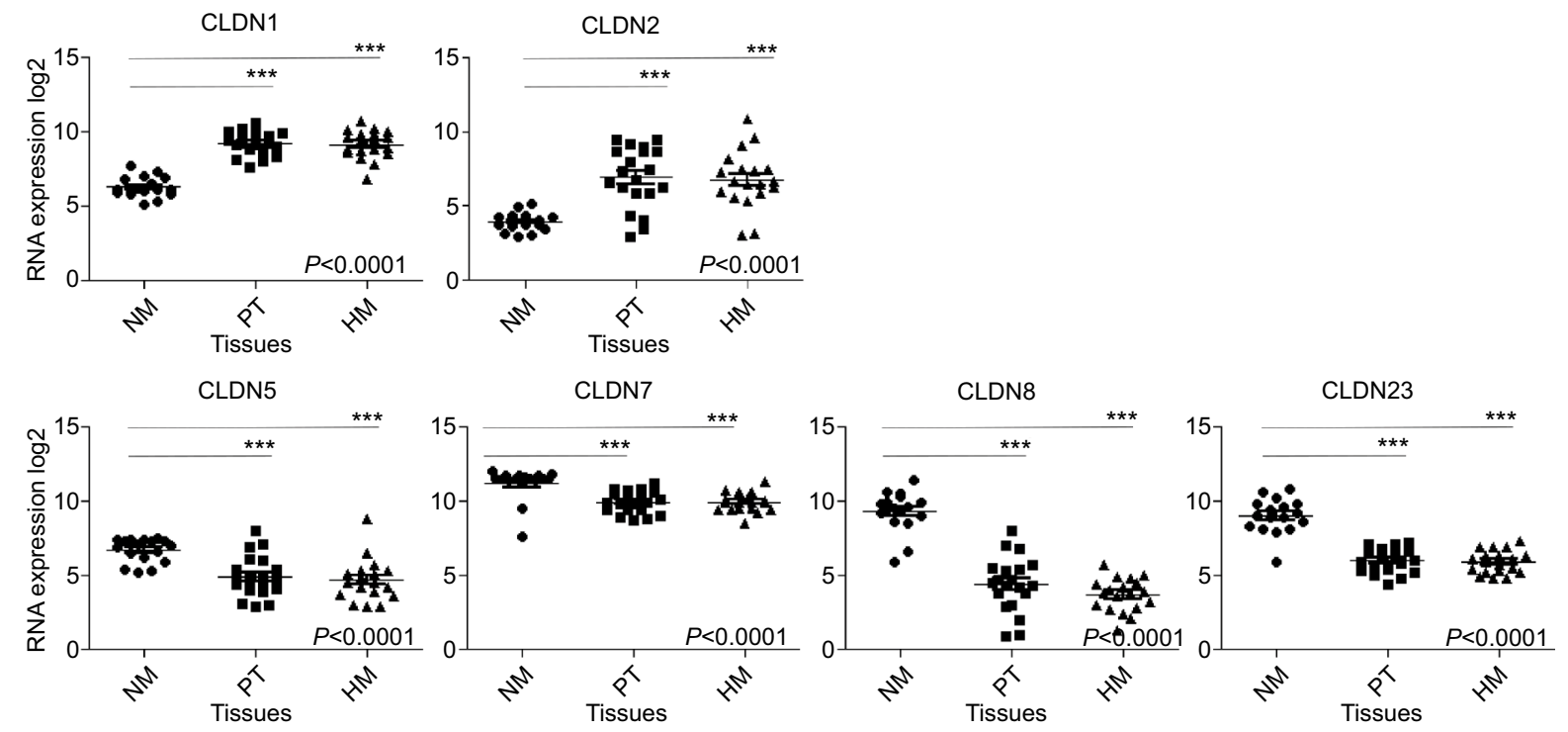

Figure I Expression of CLDN genes.

Notes: (A) Mean expression of 18 CLDN genes in NM ( $n=17)$, PT ( $n=20)$, and HM ( $n=19)$. The Affymetrix RNA expression data were log2 transformed. (B) Detailed analysis of the RNA expression levels of six CLDN genes in the NM, PT, and HM samples described in (A). Horizontal bars indicate the mean value. $* * * P<0.00 \mathrm{I}=(\mathrm{Kruskal}-\mathrm{Wallis}$ test). Abbreviations: CRC, colorectal cancer; HM, hepatic metastases; NM, normal mucosa; PT, primary CRC tumors.

\section{Association between CLDN expression level and OS and PFS}

Then, the correlation between CLDN gene expression level and PFS and OS rates was evaluated in the 143 patients divided into two groups (high/low expression relative to the median $C L D N$ gene expression) of the same size. This analysis found a significant correlation only between $C L D N 2$ gene expression (low/high) and OS $(P=0.03)$ and PFS $(P=0.005)$, and between $C L D N 4$ gene expression and $\mathrm{OS}(P=0.01$; Figure $4 \mathrm{~A}$ and $\mathrm{B})$. These results were confirmed in an independent cohort of 80 patients (Tsuji's cohort; ${ }^{44}$ Figure S4A).

As low CLDN2 expression and high CLDN4 tumor expression were good prognostic factors in patients with $\mathrm{CRC}$, the next step was to assess whether OS was correlated with the expression of both CLDNs. OS rate was significantly higher in patients with $\mathrm{CRC}$ displaying concomitant low CLDN2 and high CLDN4 expressions, particularly compared with patients with high $C L D N 2$ and low CLDN4 tumor expressions ( $P=0.006$; Figure $4 \mathrm{C}$ ). OS time was more than twofold longer in patients with low CLDN2/high CLDN4 tumors than in those with high $C L D N 2 /$ low $C L D N 4$ tumors (38 months vs 14.4 months). Moreover, analysis of the correlation between OS and CLDN2 and CLDN4 gene expressions according to the CRC molecular subtype confirmed the significant associations between CLDN2 and CLDN4 expressions and OS for the CMS3 and C3 and the CMS2 and $\mathrm{C} 1$ subtypes, respectively (Figure $\mathrm{S} 4 \mathrm{~B}$ ).

Similarly, high expressions of CLDN3 and CLDN23 and low expression of CLDN6 in the CMS2 subtype were associated with longer OS (Figure 5). Low CLDN6 expression 


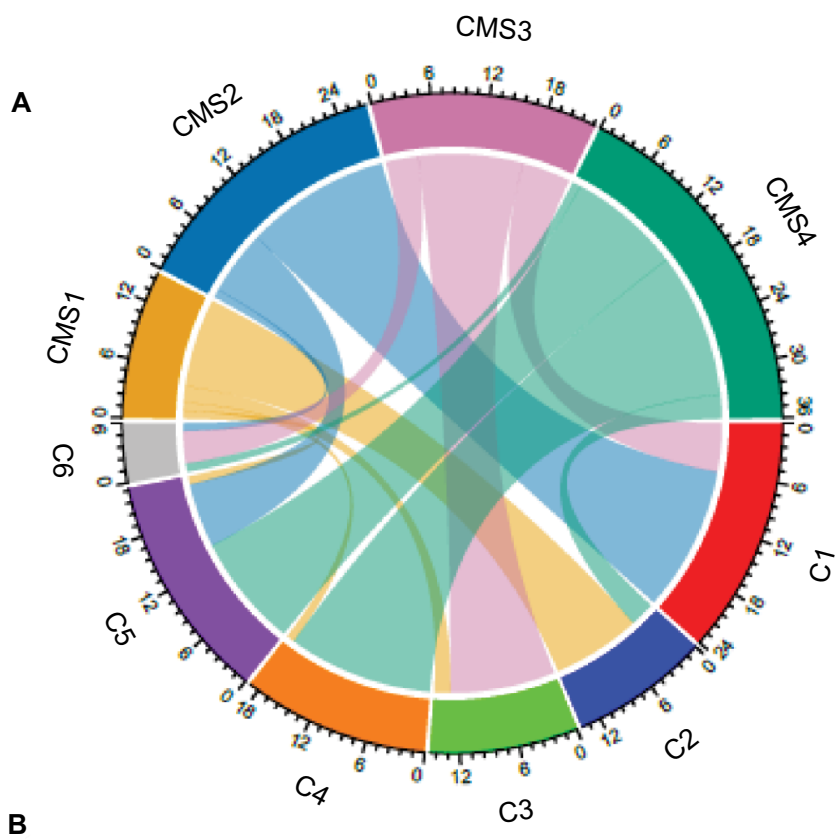

\begin{tabular}{|c|c|c|c|c|c|c|}
\hline & C1 & C2 & C3 & C4 & C5 & C6 \\
\hline CMS1 & $0 \%$ & $73 \%$ & $13 \%$ & $7 \%$ & $7 \%$ & $0 \%$ \\
\hline CMS2 & $62 \%$ & $0 \%$ & $0 \%$ & $0 \%$ & $27.5 \%$ & $0.5 \%$ \\
\hline CMS3 & $26 \%$ & $0 \%$ & $57 \%$ & $0 \%$ & $0 \%$ & $18 \%$ \\
\hline CMS4 & $0 \%$ & $8 \%$ & $0 \%$ & $50 \%$ & $39 \%$ & $3 \%$ \\
\hline
\end{tabular}

Figure 2 Interrelationships between the consensus classification (four subtypes: CMSI to 4) and the Marisa's classification (six molecular subtypes: CI to C6) of the I43 CRC samples included in this study.

Notes: (A) Chord diagram to visualize the relationships among subtypes (in different colors) of the two CRC classifications arranged radially. Data are connected to each other using arcs; each connection is proportional to the arc size. (B) Percentage of CRC samples classified as CI to C6 included in each CMS subtype.

Abbreviation: $\mathrm{CRC}$, colorectal cancer.

in the CMS2 subtype and high CLDN23 expression in the CMS2 and $\mathrm{C} 1$ subtypes were correlated with better PFS rates (Figure S5). In the CMS4 and C4 subtypes, low expression of CLDN12 and CLDN23 was associated with longer OS (Figure 5). In the $\mathrm{C} 4$ subtype, low $C L D N 11$ expression was correlated with longer OS (Figure 5) and also PFS (Figure S5). Finally, high $C L D N 8$ expression was associated with longer $\mathrm{OS}$ in the $\mathrm{C} 1$ and $\mathrm{C} 3$ subtypes (Figure 5).

\section{Discussion}

In this study, using Affymetrix gene expression data from 143 patients with CRC categorized according the CRC molecular subtypes, ${ }^{42}$ we showed that CLDN gene expression profiles vary according to the tumor stage (normal tissue, primary tumor, and metastasis) and also the molecular subtype. We assessed $C L D N$ gene expression in the four subtypes proposed by the consensus molecular classification, ${ }^{39}$ and in the six subtypes defined by Marisa et $a l^{36}$ to refine the tumor type identification. Our analysis (summary in Figure 6) indicated that eight CLDNs were differentially expressed in CRC tumors compared with normal mucosa. $C L D N$ expression alterations were mainly seen in the CMS2/C1 and CMS4/ $\mathrm{C} 4$ subtypes. The changes in the expression of these eight $C L D N$ genes were associated with a prognostic value in the whole cohort of CRC samples and also in specific molecular subtypes. Conversely, we did not detect any differential expression or prognostic value of $C L D N$ expression in the CMS1 and $\mathrm{C} 2$ subtypes. This could be due to the low number of tumors in these two groups ( $\mathrm{n}=15$ and 17 , respectively).

$C L D N$ gene expression in normal colon tissues is consistent with a previous study where the expression patterns were analyzed along the proximal-distal axis of the human intestine by real-time PCR. ${ }^{45}$ In our study, $C L D N 3,4,7,8$, and 23 displayed the highest expressions in normal colon epithelial cells with a predominant expression of CLDN7. $C L D N 3,4$, and 7 are strongly expressed in several normal tissues, including colon, and their similar expression and localization profiles suggest a coordinated regulation. ${ }^{46}$ These CLDNs have an important role in the maintenance of homeostasis of colon epithelium. CLDNs 3 and 4 have been classified as colon barrier proteins. ${ }^{47,48}$ CLDN 7 maintains the barrier function of the intestinal epithelium and regulates 
A

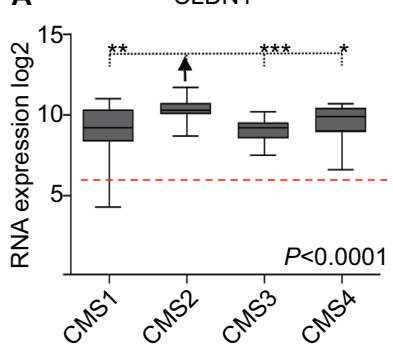

B

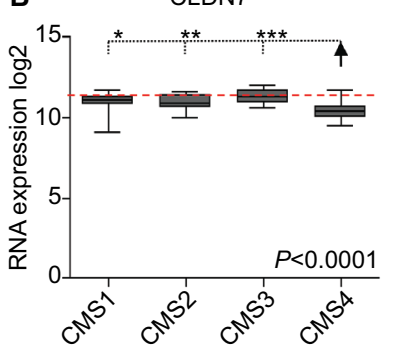

C

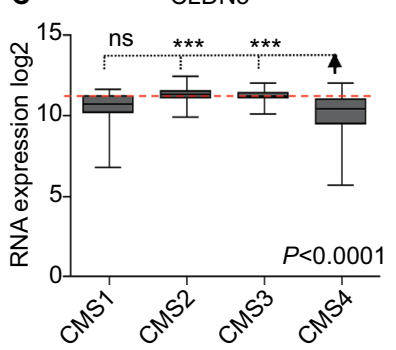

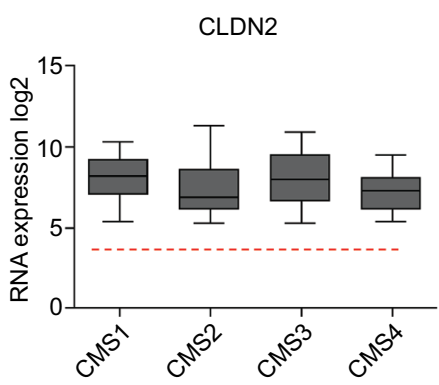
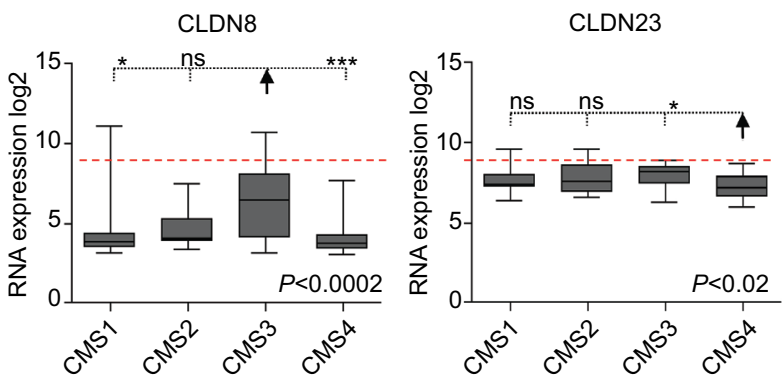

CLDN4

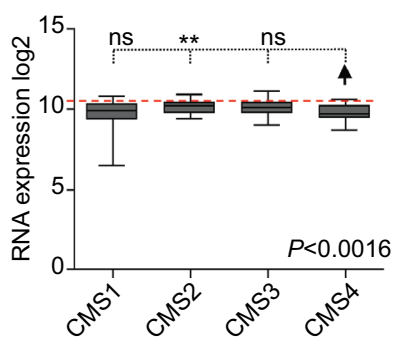

CLDN6

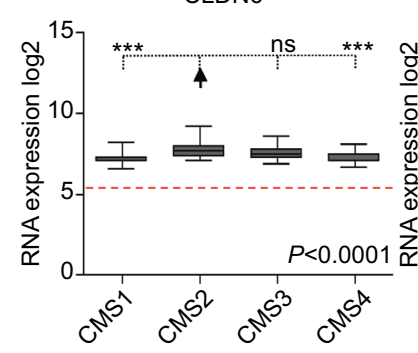

CLDN11

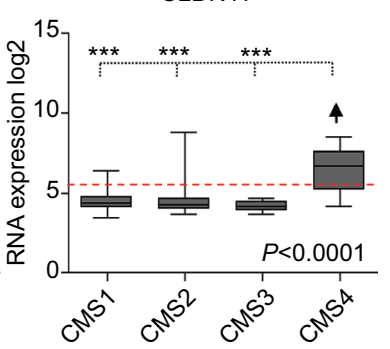

Figure 3 Differential CLDN expression in CRC samples classified using the CMS classification.

Notes: (A) CLDN genes upregulated in tumor samples compared with normal mucosa. (B) CLDN genes downregulated in tumors compared with normal mucosa. (C) CLDN genes with similar expression in normal mucosa and tumor samples. The red horizontal line indicates CLDN expression in normal mucosa within the CRC samples. The arrow highlights the subtype where CLDN expression is the most significantly different compared with the other subtypes (Kruskal-Wallis/Dunn's test). $* P<0.05$; $* * P<0.01$; $* * * P<0.001$.

Abbreviation: CRC, colorectal cancer.

epithelial cell renewal. ${ }^{49} \mathrm{CLDN} 7$ is localized at apical TJs and also in basolateral membranes, and CLDN 7-mediated cell-matrix interaction is indispensable in the intestine. ${ }^{50}$ Except for $C L D N 4$, their expression was downregulated in CRC samples. CLDN3 and CLDN7 downregulation was more pronounced in the mesenchymal CMS4 and in the stem cell phenotype-like $\mathrm{C} 4$ subtypes. CMS4 and $\mathrm{C} 4$ tumors are characterized by activation of pathways related to EMT and stemness. ${ }^{36,38,39}$ Bhat et al ${ }^{24}$ reported that CLDN7 has a key role in EMT regulation in colon epithelial cells, and that low CLDN7 expression promotes EMT and tumor progression. CLDN7 is also frequently associated with the stem cell marker EPCAM, ${ }^{51}$ and this association could contribute to EMT. ${ }^{52}$ CLDN8 displayed the strongest downregulation in CRC samples, but little is known about its function in the colon. $C L D N 8$ was identified as a critical downstream component of the IL9 inflammatory cascade in inflammatory bowel disease.$^{53}$ Here, we found that in the $\mathrm{C} 1$ and $\mathrm{C} 3$ subtypes, CLDN8 expression has a prognostic value. The common features of these two subtypes are KRAS mutations and the suppression of pathways associated with activation of the immune system and EMT. ${ }^{36}$ Moreover, higher expressions of CLDN3 and CLDN4 were associated with a better outcome in patients with CMS2 and $\mathrm{C} 1$ cancers. These findings are in agreement with CLDN3 and CLDN7 tumor-suppressive functions; $; 3,54,55$ conversely, the possible tumor suppressor role of CLDN4 and CLDN8 remains to be demonstrated.

CLDN23 expression was significantly reduced in CRC samples, as previously reported in different intestinal cancer types, ${ }^{56}$ including CRC. ${ }^{17,35,57}$ Like CLDN3, CLDN4, and $C L D N 7, C L D N 23$ was only slightly downregulated in the CMS4 and C4 subtypes, and this was correlated with longer OS. We can hypothesize that in these subtypes, CLDN23 expression is regulated by stromal suppressor 

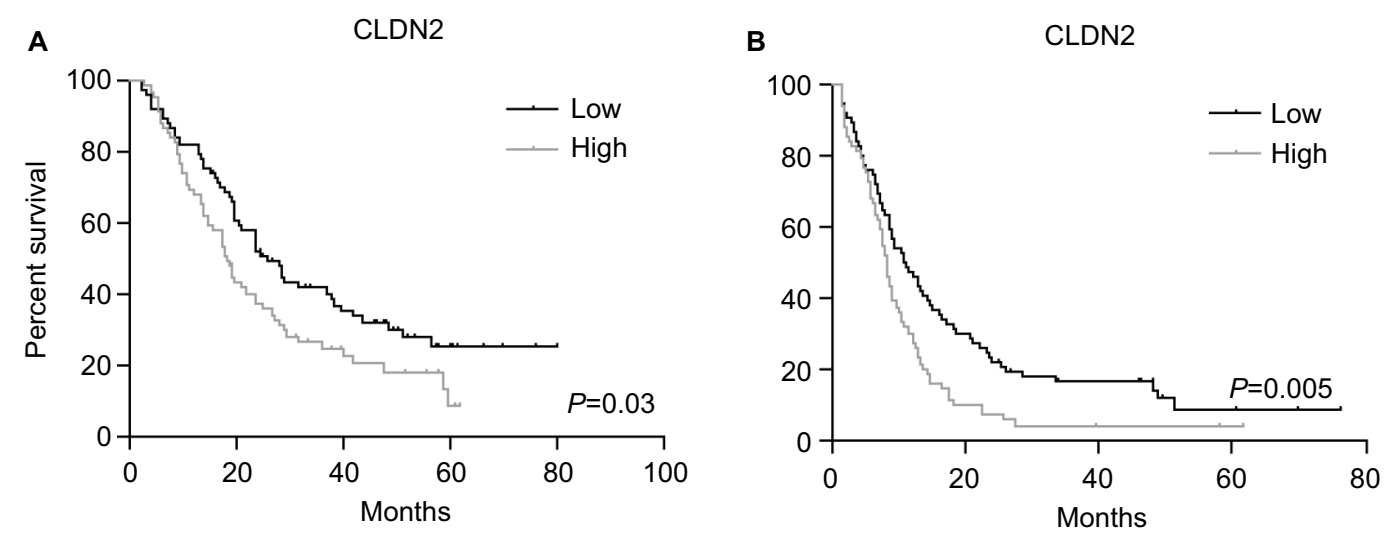

CLDN4
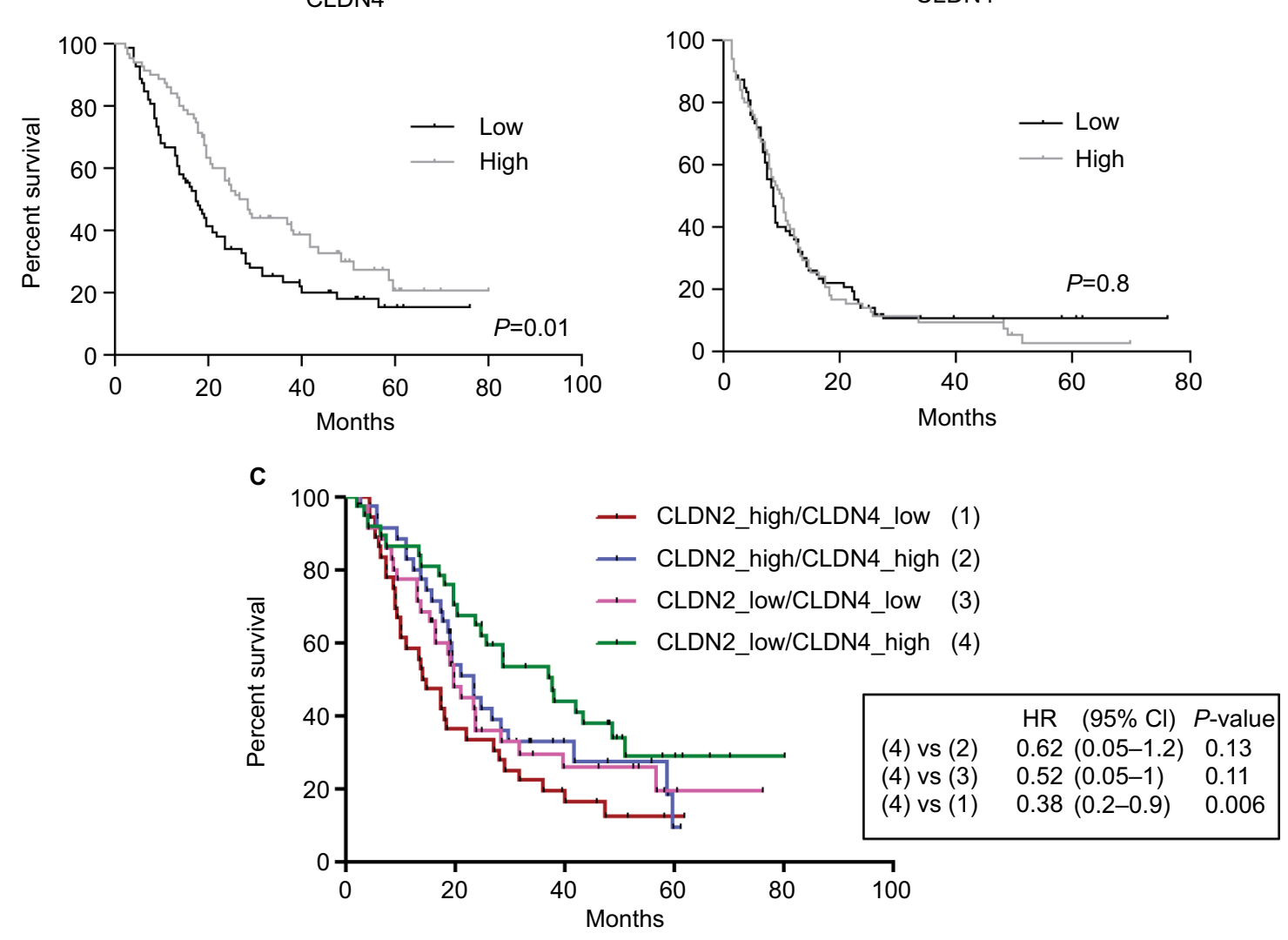

Figure 4 Clinical value of CLDN gene expression in the I 43 CRC samples.

Notes: Association between CLDN2 and CLDN4 gene expression levels and (A) OS and (B) PFS (log-rank test). High and low: higher or lower expressions relative to the median expression for that CLDN gene. (C) Association of combined CLDN2 and CLDN4 gene expressions with OS.

Abbreviations: CRC, colorectal cancer; OS, overall survival; PFS, progression-free survival.

genes. Conversely, in the canonical CMS2 and C1 subtypes, patients with low CLDN23 tumor expression had shorter OS and PFS, in agreement with a previous analysis in 53 patients with CRC. ${ }^{57} C L D N 23$ seems to have dual role as a tumor suppressor and a tumor promoter, depending on the CRC subtype where it is expressed. This hypothesis requires additional investigations because very few studies have assessed CLDN23's role in CRC since its first characteriza- tion following the observation that $C L D N 23$ is downregulated in gastric cancer. ${ }^{56}$

On the other hand, the expressions of CLDN1 and CLDN2 were significantly increased in tumor tissues, compared with normal mucosa. This confirms previous reports on their upregulation in $\mathrm{CRC}$ and their involvement in CRC cell tumorigenicity. ${ }^{22,28,58,59}$ Induction of $C L D N 1$ and $C L D N 2$ expressions has been related to overactivation 


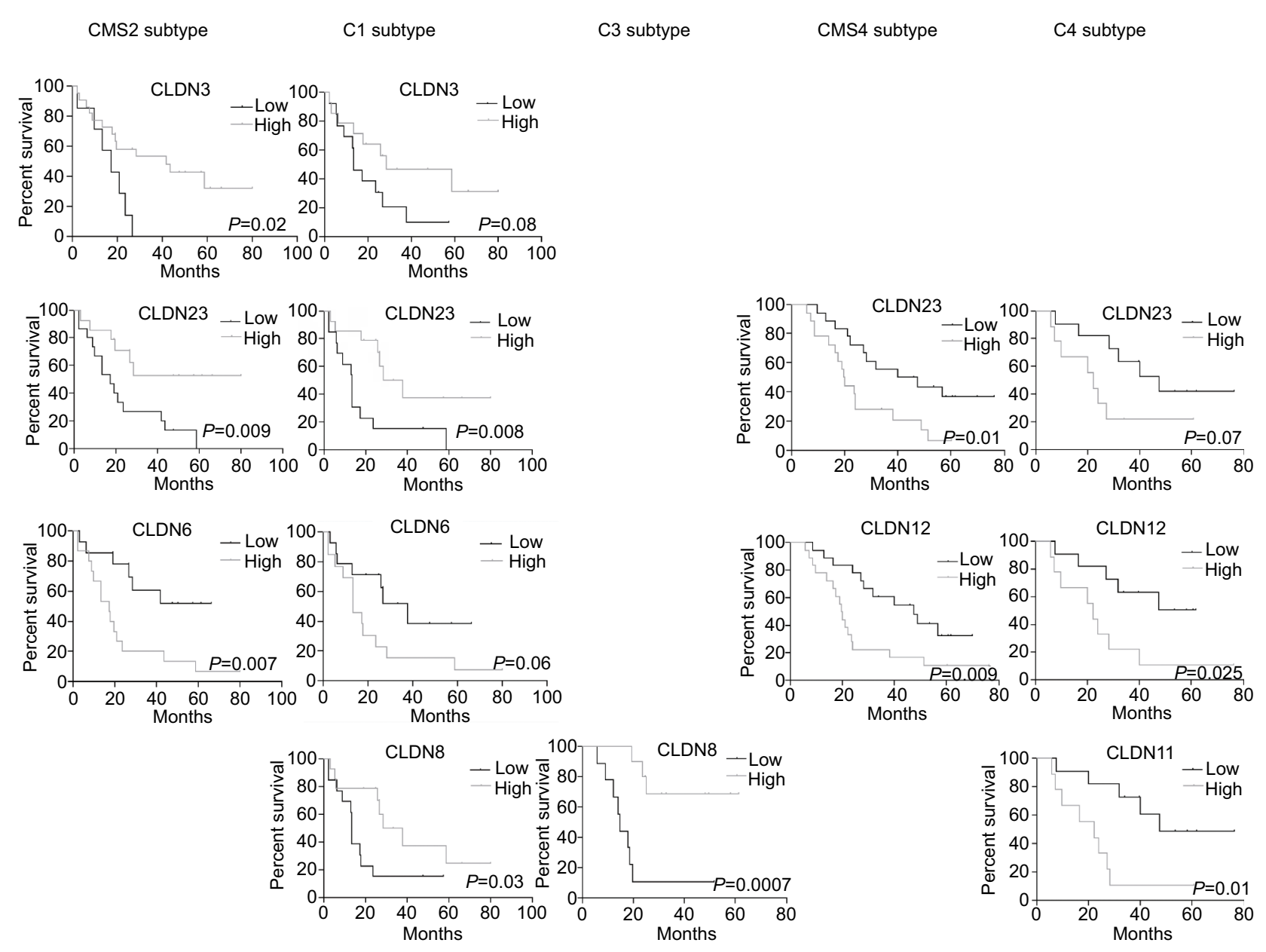

Figure 5 Association of CLDN gene expression with OS in the different CRC subtypes.

Notes: Log-rank test. High/low: expression higher/lower than the median value for that CLDN gene.

Abbreviation: CRC, colorectal cancer; OS, overall survival.

of WNT/ $\beta$-catenin signaling in CRC cells. ${ }^{60,61}$ Moreover, expressions of CLDN1 and CLDN2 have been associated with EMT and cancer progression. ${ }^{25,30}$ We previously showed that CLDN1 expression is higher in the canonical CMS2 subtype, which includes the $\mathrm{C} 1$ and $\mathrm{C} 5$ subtypes, and represents epithelial tumors with marked upregulation of WNT and MYC downstream targets, and that PFS is significantly longer in patients with $\mathrm{C} 3$ and $\mathrm{C} 5$ tumors with low CLDN1 expression. ${ }^{28}$ Here, we found that CLDN2 level is not different among CRC subtypes, but has a strong prognostic value in all patients and also in the CMS3 and C3 subtypes, which often harbor $K R A S$-activating mutations. Our findings are in agreement with recent data showing that high CLDN2 expression is linked to posttreatment recurrence in patients with stage II/III CRC. ${ }^{62}$ This makes CLDN2 a good candidate for therapeutic target in CRC.

Three other claudin genes (CLDN6, CLDN11, and $C L D N 12$ ) also displayed a prognostic value. CLDN6 was more expressed in the CMS2 and C1 subtypes, and low expression in CMS2 was associated with longer OS and PFS. CLDN6 expression and function have never been studied in CRC. It was described as a cancer-promoting factor in gastric cancer, ${ }^{63}$ and as a tumor suppressor in breast cancer. ${ }^{64}$ Low CLDN11 expression correlation with longer OS and PFS in the $\mathrm{C} 4$ subtype (at high risk of relapse), and CLDN11 upregulation in the CMS4 and $\mathrm{C} 4$ subtypes suggest a protumor function in CRC. CLDN11 could play a role in the TGF $\beta 1-O C L N / C L D N 11$ paracrine axis between cancer cells and cancer-associated fibroblasts in CRC. ${ }^{65}$ CLDN12 was strongly expressed in both normal and tumor colon tissues. In the CMS4 and C4 subtypes, low expression was associated with better prognosis. CLDN12 contributes to $\mathrm{Ca}^{2+}$ absorption in intestinal epithelial cells, ${ }^{66}$ and it is one of the few claudins that do not possess a PDZ binding motif. ${ }^{7}$

The specific biological features of the CRC subtypes could explain the differences in $C L D N$ gene expression. 

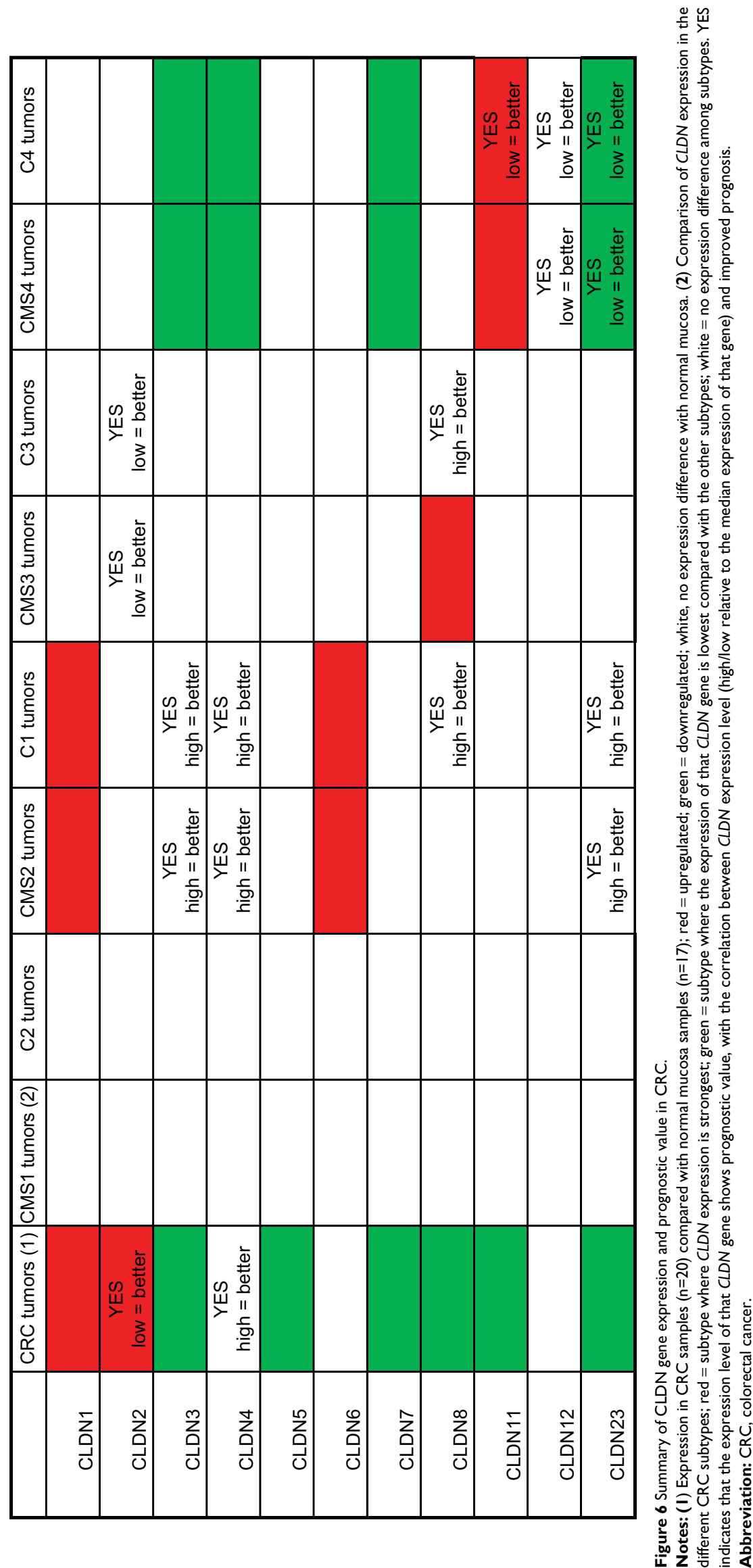
Indeed, the gene expression profiles of claudins involved in EMT (CLDN7), acting as tumor suppressors (CLDN3, 7, 23) or linked to the stem cell phenotype (CLDN7), were markedly different in CMS4/C4 tumors (Figure 6). In CMS2/C1 and C5 tumors, $C L D N$ gene expression seemed to be more dependent on WNT signaling, as already described for CLDN1.

$C L D N$ gene downregulation could be explained by alterations in their sequences or in epigenetic regulation mechanisms, such as histone modifications, DNA methylation, and chromatin remodeling. This was demonstrated for CLDN1, ${ }^{16}$ CLDN2, ${ }^{18}$ CLDN7, ${ }^{19}$ and CLDN23. ${ }^{17}$

Changes in CLDN gene expression were essentially seen in the CMS2 and CMS4 subtypes for which a clear distinction in sensitivity to chemotherapy-induced apoptosis has been reported. ${ }^{67}$ This suggests that in CRC, claudins could play a role in chemoresistance, as previously shown for CLDN4 and CLDN7 in ovarian cancer, ${ }^{68,69}$ and more recently, for CLDN6 in triple-negative breast cancer cells. ${ }^{70}$

Altogether, we showed that $C L D N$ gene expression has a significant clinical relevance. First, in the whole sample, the expressions of CLDN2 and CLDN4 alone showed a strong prognostic value that was increased when both genes were associated (median survival: 14.41 months for patients with high $C L D N 2 /$ low CLDN4 expression and 38 months for patients with low CLDN2/high CLDN4 expression; HR $=0.38$ ). If these expression data are confirmed at the protein level, a prognostic gene/protein expression score can be developed for CRC patients. Second, for almost all CRC subtypes, we identified at least one $C L D N$ gene, the expression of which was correlated with survival (Figure 6). In addition, we defined a CMS4/C4 tumor subtype signature characterized by low $C L D N 3,4,7$, and 23 expressions associated with high $C L D N 11$ expression. Moreover, in the C1 subtype, high $C L D N 3,4,8$, and 23 expressions could be associated with good prognosis. In a breast cancer subtype, a signature characterized by low CLDN gene expression was associated with an aggressive phenotype. ${ }^{71}$ Finally, the best candidates as therapeutic targets seem to be CLDN6 in CMS2 tumors and CLDN11 in C4 tumors because they are overexpressed in these subtypes and low expression was associated with better prognosis. Future studies should thoroughly investigate their value as prognostic markers or therapeutic targets.

\section{Conclusion}

$C L D N$ gene expression differences in CRC reflect $\mathrm{CRC}$ heterogeneity, and indicate that it is not enough simply to examine $C L D N$ expression level globally. Moreover, claudin functions are not limited to TJs. Indeed, growing evidence shows that claudins are localized to sites outside the TJ complex, ${ }^{72}$ or even delocalized from the membrane to the nucleus, thus acquiring an important role in tumorigenesis. Finally, our findings demonstrated that CLDN expression is regulated in a CRC molecular subtype manner, and highlighted that to evaluate claudin function and potential value as prognostic markers or therapeutic targets, it is essential to take into account $\mathrm{CRC}$ heterogeneity.

\section{Data sharing statement}

Transcript profiles: GSE62080, GSE72970, GSE28702.

\section{Acknowledgments}

This work was supported by grants from the French National Research Agency under the program "Investissementsd'avenir", Grant Agreement LabExMAbImprove: ANR-10-LABX-53, and SIRIC Montpellier Cancer Grant: "INCa-DGOS-Inserm 6045".

\section{Disclosure}

The authors report no conflicts of interest in this work.

\section{References}

1. Royer C, Lu X. Epithelial cell polarity: a major gatekeeper against cancer? Cell Death Differ. 2011;18(9):1470-1477.

2. Anderson JM, Van Itallie CM. Physiology and function of the tight junction. Cold Spring Harb Perspect Biol. 2009;1(2):a002584.

3. Colegio OR, Van Itallie CM, McCrea HJ, Rahner C, Anderson JM. Claudins create charge-selective channels in the paracellular pathway between epithelial cells. Am J Physiol Cell Physiol. 2002;283(1):C142-C147.

4. Oshima T, Miwa H. Gastrointestinal mucosal barrier function and diseases. J Gastroenterol. 2016;51(8):768-778.

5. Van Itallie CM, Anderson JM. Architecture of tight junctions and principles of molecular composition. Semin Cell Dev Biol. 2014;36:157-165.

6. Tsukita S, Furuse M. Pores in the wall: claudins constitute tight junction strands containing aqueous pores. J Cell Biol. 2000;149(1):13-16.

7. Günzel D, Yu AS. Claudins and the modulation of tight junction permeability. Physiol Rev. 2013;93(2):525-569.

8. Lal-Nag M, Morin PJ. The claudins. Genome Biol. 2009;10(8):235-235.

9. Chen J, Zhang M. The Par3/Par6/aPKC complex and epithelial cell polarity. Exp Cell Res. 2013;319(10):1357-1364.

10. Tsukita S, Furuse M, Itoh M. Multifunctional strands in tight junctions. Nat Rev Mol Cell Biol. 2001;2(4):285-293.

11. Singh AB, Sharma A, Dhawan P. Claudin family of proteins and cancer: an overview. J Oncol. 2010;2010:541957.

12. Van Itallie CM, Anderson JM. Claudin interactions in and out of the tight junction. Tissue Barriers. 2013;1(3):e25247.

13. Osanai M, Takasawa A, Murata M, Sawada N. Claudins in cancer: bench to bedside. Pflugers Arch. 2017;469(1):55-67.

14. Singh AB, Uppada SB, Dhawan P. Claudin proteins, outside-in signaling, and carcinogenesis. Pflugers Arch. 2017;469(1):69-75.

15. Garcia-Hernandez V, Quiros M, Nusrat A. Intestinal epithelial claudins: expression and regulation in homeostasis and inflammation. Ann NY Acad Sci. 2017;1397(1):66-79.

16. Krishnan M, Singh AB, Smith JJ, et al. HDAC inhibitors regulate claudin-1 expression in colon cancer cells through modulation of mRNA stability. Oncogene. 2010;29(2):305-312. 
17. Maryan N, Statkiewicz M, Mikula M, et al. Regulation of the expression of claudin 23 by the enhancer of zeste 2 polycomb group protein in colorectal cancer. Mol Med Rep. 2015;12(1):728-736.

18. Ahmad R, Kumar B, Pan K, Dhawan P, Singh AB. HDAC-4 regulates claudin-2 expression in EGFR-ERK1/2 dependent manner to regulate colonic epithelial cell differentiation. Oncotarget. 2017;8(50):87718-87736.

19. Nakayama F, Semba S, Usami Y, Chiba H, Sawada N, Yokozaki H. Hypermethylation-modulated downregulation of claudin-7 expression promotes the progression of colorectal carcinoma. Pathobiology. 2008;75(3):177-185.

20. Tabariès S, Siegel PM. The role of claudins in cancer metastasis. Oncogene. 2017;36(9):1176-1190.

21. Chao YC, Pan SH, Yang SC, et al. Claudin-1 is a metastasis suppressor and correlates with clinical outcome in lung adenocarcinoma. Am J Respir Crit Care Med. 2009;179(2):123-133.

22. Dhawan P, Singh AB, Deane NG. Claudin-1 regulates cellular transformation and metastatic behavior in colon cancer. $J$ Clin Invest. 2005;115(7):1765-1776.

23. Joyce T, Cantarella D, Isella C, Medico E, Pintzas A. A molecular signature for epithelial to mesenchymal transition in a human colon cancer cell system is revealed by large-scale microarray analysis. Clin Exp Metastasis. 2009;26(6):569-587.

24. Bhat AA, Pope JL, Smith JJ, et al. Claudin-7 expression induces mesenchymal to epithelial transformation (MET) to inhibit colon tumorigenesis. Oncogene. 2015;34(35):4570-4580.

25. Bhat AA, Ahmad R, Uppada SB, Singh AB, Dhawan P. Claudin-1 promotes TNF- $\alpha$-induced epithelial-mesenchymal transition and migration in colorectal adenocarcinoma cells. Exp Cell Res. 2016;349(1): $119-127$.

26. Singh AB, Dhawan P. Claudins and cancer: fall of the soldiers entrusted to protect the gate and keep the barrier intact. Semin Cell Dev Biol. 2015;42:58-65.

27. Jiang L, Yang L, Huang H, Liu BY, Zu G. Prognostic and clinical significance of claudin-1 in colorectal cancer: a systemic review and meta-analysis. Int J Surg. 2017;39:214-220.

28. Cherradi S, Ayrolles-Torro A, Vezzo-Vié N, et al. Antibody targeting of claudin-1 as a potential colorectal cancer therapy. J Exp Clin Cancer Res. 2017;36(1):89.

29. Ouban A. Claudin-1 role in colon cancer: an update and a review. Histol Histopathol. 2018;33(10):1013-1019.

30. Dhawan P, Ahmad R, Chaturvedi R, et al. Claudin-2 expression increases tumorigenicity of colon cancer cells: role of epidermal growth factor receptor activation. Oncogene. 2011;30(29):3234-3247.

31. Buchert M, Papin M, Bonnans C. Symplekin promotes tumorigenicity by up-regulating claudin-2 expression. Proc Natl Acad Sci U S A. 2010;107(6):2628-2633.

32. Ding L, Wang L, Sui L, et al. Claudin-7 indirectly regulates the integrin/ FAK signaling pathway in human colon cancer tissue. J Hum Genet 2016;61(8):711-720

33. Darido C, Buchert M, Pannequin J, et al. Defective claudin-7 regulation by Tcf- 4 and Sox-9 disrupts the polarity and increases the tumorigenicity of colorectal cancer cells. Cancer Res. 2008;68(11):4258-4268.

34. Wang X, Tully O, Ngo B, Zitin M, Mullin JM. Epithelial tight junctional changes in colorectal cancer tissues. ScientificWorldJournal. 2011;11:826-841.

35. Bujko M, Kober P, Mikula M, Ligaj M, Ostrowski J, Siedlecki JA. Expression changes of cell-cell adhesion-related genes in colorectal tumors. Oncol Lett. 2015;9(6):2463-2470.

36. Marisa L, de Reyniès A, Duval A, et al. Gene expression classification of colon cancer into molecular subtypes: characterization, validation, and prognostic value. PLoS Med. 2013;10(5):e1001453.

37. Sadanandam A, Lyssiotis CA, Homicsko K, et al. A colorectal cancer classification system that associates cellular phenotype and responses to therapy. Nat Med. 2013;19(5):619-625.
38. Dienstmann R, Vermeulen L, Guinney J, Kopetz S, Tejpar S, Tabernero J. Consensus molecular subtypes and the evolution of precision medicine in colorectal cancer. Nat Rev Cancer. 2017;17(2):79-92.

39. Guinney J, Dienstmann R, Wang X, et al. The consensus molecular subtypes of colorectal cancer. Nat Med. 2015;21(11):1350-1356.

40. Del Rio M, Molina F, Bascoul-Mollevi C, et al. Gene expression signature in advanced colorectal cancer patients select drugs and response for the use of leucovorin, fluorouracil, and irinotecan. J Clin Oncol. 2007;25(7):773-780.

41. Del Rio M, Mollevi C, Vezzio-Vie N, Bibeau F, Ychou M, Martineau P. Specific extracellular matrix remodeling signature of colon hepatic metastases. PLoS One. 2013;8(9):e74599.

42. Del Rio M, Mollevi C, Bibeau F, et al. Molecular subtypes of metastatic colorectal cancer are associated with patient response to irinotecanbased therapies. Eur J Cancer. 2017;76:68-75.

43. Copois V, Bibeau F, Bascoul-Mollevi C, et al. Impact of RNA degradation on gene expression profiles: assessment of different methods to reliably determine RNA quality. J Biotechnol. 2007;127(4):549-559.

44. Tsuji S, Midorikawa Y, Takahashi T, et al. Potential responders to FOLFOX therapy for colorectal cancer by Random Forests analysis. Br J Cancer. 2012;106(1):126-132.

45. Lameris AL, Huybers S, Kaukinen K, et al. Expression profiling of claudins in the human gastrointestinal tract in health and during inflammatory bowel disease. Scand J Gastroenterol. 2013;48(1):58-69.

46. Hewitt KJ, Agarwal R, Morin PJ. The claudin gene family: expression in normal and neoplastic tissues. BMC Cancer. 2006;6:186.

47. Milatz S, Krug SM, Rosenthal R, et al. Claudin-3 acts as a sealing component of the tight junction for ions of either charge and uncharged solutes. Biochim Biophys Acta. 1798;2010(11):2048-2057.

48. Hering NA, Andres S, Fromm A, et al. Transforming growth factor- $\beta$, a whey protein component, strengthens the intestinal barrier by upregulating claudin-4 in HT-29/B6 cells. J Nutr. 2011;141(5):783-789.

49. Tanaka H, Takechi M, Kiyonari H, Shioi G, Tamura A, Tsukita S. Intestinal deletion of claudin-7 enhances paracellular organic solute flux and initiates colonic inflammation in mice. Gut. 2015;64(10):1529-1538.

50. Ding $\mathrm{L}, \mathrm{Lu} Z$, Foreman $\mathrm{O}$, et al. Inflammation and disruption of the mucosal architecture in claudin-7-deficient mice. Gastroenterology. 2012;142(2):305-315

51. Kuhn S, Koch M, Nübel T, et al. A complex of EpCAM, claudin-7, CD44 variant isoforms, and tetraspanins promotes colorectal cancer progression. Mol Cancer Res. 2007;5(6):553-567.

52. Philip R, Heiler S, Mu W, Büchler MW, Zöller M, Thuma F. Claudin-7 promotes the epithelial-mesenchymal transition in human colorectal cancer. Oncotarget. 2015;6(4):2046-2063.

53. Li L, Huang S, Wang H, et al. Cytokine IL9 triggers the pathogenesis of inflammatory bowel disease through the miR21-CLDN8 pathway. Inflamm Bowel Dis. 2018:24(10):2211-2223.

54. Ahmad R, Kumar B, Chen Z, et al. Loss of claudin-3 expression induces IL6/gp130/Stat3 signaling to promote colon cancer malignancy by hyperactivating $\mathrm{Wnt} / \beta$-catenin signaling. Oncogene. 2017;36(47):6592-6604

55. Oshima T, Kunisaki C, Yoshihara K, et al. Reduced expression of the claudin-7 gene correlates with venous invasion and liver metastasis in colorectal cancer. Oncol Rep. 2008;19(4):953-959.

56. Katoh M, Katoh M. CLDN23 gene, frequently down-regulated in intestinal-type gastric cancer, is a novel member of CLAUDIN gene family. Int J Mol Med. 2003;11(6):683-689.

57. Pitule P, Vycital O, Bruha J, et al. Differential expression and prognostic role of selected genes in colorectal cancer patients. Anticancer Res 2013;33(11):4855-4865.

58. Kinugasa T, Huo Q, Higashi D, et al. Selective up-regulation of claudin-1 and claudin-2 in colorectal cancer. Anticancer Res. 2007;27(6A):3729-3734.

59. Aung PP, Mitani Y, Sanada Y, Nakayama H, Matsusaki K, Yasui W. Differential expression of claudin-2 in normal human tissues and gastrointestinal carcinomas. Virchows Arch. 2006;448(4):428-434. 
60. Miwa N, Furuse M, Tsukita S, Niikawa N, Nakamura Y, Furukawa Y. Involvement of claudin-1 in the beta-catenin/Tcf signaling pathway and its frequent upregulation in human colorectal cancers. Oncol Res. 2001;12(11-12):469-476.

61. Mankertz J, Hillenbrand B, Tavalali S, Huber O, Fromm M, Schulzke JD. Functional crosstalk between Wnt signaling and Cdx-related transcriptional activation in the regulation of the claudin-2 promoter activity. Biochem Biophys Res Commun. 2004;314(4):1001-1007.

62. Paquet-Fifield S, Koh SL, Cheng L, et al. Tight junction protein claudin-2 promotes self-renewal of human colorectal cancer stem-like cells. Cancer Res. 2018;78(11):2925-2938.

63. Zavala-Zendejas VE, Torres-Martinez AC, Salas-Morales B, Fortoul TI, Montaño LF, Rendon-Huerta EP. Claudin-6, 7, or 9 overexpression in the human gastric adenocarcinoma cell line AGS increases its invasiveness, migration, and proliferation rate. Cancer Invest. 2011;29(1):1-11.

64. Wu Q, Liu Y, Ren Y, et al. Tight junction protein, claudin-6, downregulates the malignant phenotype of breast carcinoma. Eur J Cancer Prev. 2010;19(3):186-194.

65. Karagiannis GS, Schaeffer DF, Cho CK, et al. Collective migration of cancer-associated fibroblasts is enhanced by overexpression of tight junction-associated proteins claudin-11 and occludin. Mol Oncol. 2014;8(2):178-195.
66. Fujita $\mathrm{H}$, Sugimoto $\mathrm{K}$, Inatomi S, et al. Tight junction proteins claudin-2 and -12 are critical for vitamin D-dependent $\mathrm{Ca} 2+$ absorption between enterocytes. Mol Biol Cell. 2008;19(5):1912-1921.

67. Linnekamp JF, Hooff SRV, Prasetyanti PR, et al. Consensus molecular subtypes of colorectal cancer are recapitulated in in vitro and in vivo models. Cell Death Differ. 2018;25(3):616-633.

68. Yoshida H, Sumi T, Zhi X, Yasui T, Honda K, Ishiko O. Claudin-4: a potential therapeutic target in chemotherapy-resistant ovarian cancer. Anticancer Res. 2011;31(4):1271-1277.

69. Kim CJ, Lee JW, Choi JJ, et al. High claudin-7 expression is associated with a poor response to platinum-based chemotherapy in epithelial ovarian carcinoma. Eur J Cancer. 2011;47(6):918-925.

70. Yang M, Li Y, Ruan Y, et al. CLDN6 enhances chemoresistance to ADM via AF-6/ERKs pathway in TNBC cell line MDAMB231. Mol Cell Biochem. 2018;443(1-2):169-180.

71. Herschkowitz JI, Simin K, Weigman VJ, et al. Identification of conserved gene expression features between murine mammary carcinoma models and human breast tumors. Genome Biol. 2007;8(5):R76.

72. Hagen SJ. Non-canonical functions of claudin proteins: beyond the regulation of cell-cell adhesions. Tissue Barriers. 2017;5(2):e1327839.
Cancer Management and Research

\section{Publish your work in this journal}

Cancer Management and Research is an international, peer-reviewed open access journal focusing on cancer research and the optimal use of preventative and integrated treatment interventions to achieve improved outcomes, enhanced survival and quality of life for the cancer patient. The manuscript management system is completely online and includes

\section{Dovepress}

a very quick and fair peer-review system, which is all easy to use. Visit http://www.dovepress.com/testimonials.php to read real quotes from published authors. 